\title{
Proton Transfer from a Photoacid to Water: First Principles Simulations and Fast Fluorescence Spectroscopy
}

\author{
Alice R. Walker, ${ }^{1,2,3}$ Boning Wu ${ }^{1, \#}$, Jan Meisner, ${ }^{1,2,3}$ Michael D. Fayer, ${ }^{1, \dagger}$ and \\ Todd J. Martínez, ${ }^{1,2,3, \$}$ \\ ${ }^{1}$ Department of Chemistry, Stanford University, Stanford, CA 94305 \\ ${ }^{2}$ The PULSE Institute, Stanford University, Stanford, CA 94305 \\ ${ }^{3}$ SLAC National Accelerator Laboratory, Menlo Park, CA 94025
}

\begin{abstract}
Proton transfer reactions are ubiquitous in chemistry, especially in aqueous solutions. We investigate photo-induced proton transfer between the photoacid 8-hydroxypyrene-1,3,6trisulfonate (HPTS) and water using fast fluorescence spectroscopy and ab initio molecular dynamics simulations. Photo-excitation causes rapid proton release from the HPTS hydroxyl. Previous experiments on HPTS/water described the progress from photoexcitation to proton diffusion using kinetic equations with two time constants. The shortest time constant has been interpreted as protonated and photoexcited HPTS evolving into an "associated" state, where the proton is "shared" between the HPTS hydroxyl and an originally hydrogen bonded water. The longer time constant has been interpreted as indicating evolution to a "solvent separated" state where the shared proton undergoes long distance diffusion. In this work, we refine the previous experimental results using very pure HPTS. We then use excited state $a b$ initio molecular dynamics to elucidate the detailed molecular mechanism of aqueous excited state proton transfer in HPTS. We find that the initial excitation results in rapid rearrangement of water, forming a strong hydrogen bonded network (a "water wire") around HPTS. HPTS then deprotonates in $\leq 3 \mathrm{ps,}$ resulting in a proton that migrates back and forth along the wire before localizing on a single water molecule. We find a near linear relationship between emission wavelength and proton-HPTS distance over the simulated time scale, suggesting that emission wavelength can be used as a ruler for proton distance. Our simulations reveal that the "associated" state corresponds to a water wire with a mobile proton and that the diffusion of the proton away from this water wire (to a generalized "solvent-separated" state) corresponds to the longest experimental time constant.
\end{abstract}

\footnotetext{
\# Present address: CAS Dalian Institute of Chemical Physics, Dalian, PR China

† phone: 650 723-4446; email: fayer@ stanford.edu

¥ phone: 650 736-8860; email: toddjmartinez@gmail.com
}

Walker, et al. - Proton Transfer in HPTS - Page 1 


\section{Introduction}

Proton transfer reactions in aqueous solutions are important in many physical, chemical, and biological processes, including water oxidation, ${ }^{1}$ tautomerization of bases in DNA, ${ }^{2}$ ATP activities in living cells, ${ }^{3}$ and proton diffusion in water. ${ }^{4-5}$ Water's ability to form complex hydrogen bonding networks, particularly in the presence of ions, gives rise to various suggested mechanisms for accelerated proton transfer, including stepwise hopping and collective deprotonation within a water wire. ${ }^{6-8}$ A useful approach to study such processes is to monitor the photo-induced proton transfer between an excited photoacid and water. ${ }^{9-10}$ A photoacid is a particular type of molecule that becomes a stronger acid after promotion to an electronic excited state. A common photoacid is 8-hydroxypyrene-1,3,6-trisulfonate (pyranine, or HPTS), which has a pKa near 7 in the ground state but drops to $\sim 0$ upon photoexcitation. ${ }^{11}$ The proton transfer between HPTS and water has been extensively studied with time resolved fluorescence, transient IR/visible absorption, and stimulated Raman spectroscopies. ${ }^{12-19}$

Upon the photo-excitation of an HPTS molecule in water, several fast and slow processes have been observed by transient absorption and time resolved fluorescence spectroscopies. ${ }^{13,18-21}$ The fastest process is the Stokes shift, which takes place between a few hundred femtoseconds and a picosecond. ${ }^{13,17}$ This Stokes shift is not directly related to proton transfer. Following the Stokes shift, the observed excited state dynamics have been analyzed using kinetic equations. ${ }^{13}$ Three spectroscopically identifiable excited HPTS states have been discussed in relation to the kinetic model. The first proposed state is the "protonated state." This is the short-lived state that is initially formed after excitation. The HPTS is excited but the system of HPTS and water still essentially has its ground state configuration, including the hydroxyl proton. The hydroxyl proton in the ground state is assumed to be hydrogen bonded to an oxygen of a water molecule. The second proposed state is referred to as the "associated state." In this state the proton is assumed to have 
moved some distance from the hydroxyl oxygen towards the oxygen of the hydrogen bonded water molecule, i.e. the proton is shared between HPTS and the water molecule. The time constant of the transition from protonated state to associated state is $\sim 3 \mathrm{ps}$. The third proposed state is the deprotonated or solvent separated state. In this state, a water molecule is hydrogen bonded to the $\mathrm{O}^{-}$of deprotonated HPTS. The state of the proton is not defined other than it is no longer shared between the HPTS and the oxygen of the initially hydrogen bonded water. The time constant of the transition from the associated to deprotonated state is $\sim 90 \mathrm{ps}$. In this work, we present computational evidence demonstrating that the proposed associated state is more likely a deprotonation event where the proton remains in a water wire emanating from the HPTS molecule.

Different states of HPTS display different fluorescence spectra, where the peak maximum can be shifted by up to $80 \mathrm{~nm} \cdot{ }^{21}$ In addition to these relatively short times, there is a much slower process associated with long range proton diffusion followed by proton recombination, which reforms the associated state. The recombination occurs over nanoseconds to tens of nanoseconds. By repopulating the associated state to a small extent, the fluorescence intensity of the "associated" peak decays non-exponentially with an $\sim 90$ ps time constant. ${ }^{12,21}$ It has been shown theoretically and experimentally that the recombination causes the final complete decay of the associated state to occur as a power law, $t^{-\alpha}$, where $\alpha=\sim 1.5 .^{22-23}$ This process has been discussed in detail using a Smoluchowski type diffusion-reaction theory. ${ }^{12}$

Bulk water forms and reforms hydrogen bonds continually, with fast hydrogen bond randomization on the order of 2 ps in bulk water. ${ }^{24}$ This can change at interfaces and around solutes, which can organize the water differently than bulk water. In the case of reorganizing around an excited dye, such as HPTS, water generally rearranges on the order of a single picosecond. ${ }^{6}$ A number of theoretical studies have shown that a positive ion in water can have varying rates of 
diffusion based on the relative orientation of waters and solutes, and complex collective motions through tightly bound water wires. ${ }^{6-7}$ Rates of proton diffusion are correlated to this relative organization, with various outcomes depending on the orientations of the water molecules through the bulk..$^{25}$

The focus of this paper is to understand the nature of the associated state and what deprotonation (solvent separation) means in terms of the experimental spectral observables on the quantum molecular level. To this end we have performed ab initio molecular dynamics simulations to study the fastest dynamical events. The simulations calculate the electronic excited state energies and dynamic changes associated with the different stages of deprotonation. These are compared to the results of time dependent fast fluorescence experiments monitoring the fluorescence spectra of HPTS in aqueous solution following photo-excitation. The experiments use time correlated single photon counting, as has been described previously. ${ }^{26-27}$ The emphasis of the experiments presented here is to obtain very accurate time dependent results. In past experiments, the spectra have been somewhat contaminated by emission from a fluorescent impurity that is present in commercial HPTS. The impurity fluorescence spectrum needed to be characterized and removed from the HPTS spectra, which is difficult as its molecular identity is unknown. It needed to be modeled and subtracted. Here we avoid this step by using HPTS synthesized by Dr. Ernst Koller using a new procedure that does not produce the impurity.

For the first time, the $a b$ initio molecular dynamics simulations (including as many as 100 explicit quantum mechanical water molecules capable of proton transfer) address the details of the proton dynamics as a proton leaves the HPTS in pure water. The initial excitation results in immediate (within $100 \mathrm{fs}$ ) strengthening (or in some cases formation) of a hydrogen bond between the HPTS hydroxyl group and the nearest water molecule. This is quickly followed (200-2000 fs)

Walker, et al. - Proton Transfer in HPTS - Page 4 
by water rearrangement to form a water wire. Previous theoretical work on excited HPTS in both aqueous $^{28}$ and aqueous/acetate ${ }^{29}$ solution also observed an initial tightening of the hydrogen bond between HPTS and a water molecule coordinating to the HPTS hydroxyl group. However, the formation of a water wire was not reported, likely because most or all of the surrounding water molecules were treated with an empirical force field that restricted proton transfer. The water wire network we observe generally comprises 3-6 waters, and extends from the HPTS hydroxyl group to the nearest sulfate group that it is pointing towards. Within $3 \mathrm{ps,} \mathrm{HPTS} \mathrm{deprotonates} \mathrm{and} \mathrm{the}$ proton rapidly moves along the water wire. The overall dynamics of the proton transfer are similar to previously reported theoretical work for protons transferring through bulk water-the movement of the proton through the wire is the result of a complex collective motion with rest and burst phases, and varying degrees of proton sharing throughout the wire prior to the burst deprotonation event. ${ }^{7}$ After this burst of activity, the proton localizes on one water molecule. The emission wavelength in these stages is linear in the proton-HPTS distance, suggesting that the spectral evolution occurring on a 3 ps timescale (after an initial ultrafast Stokes shift) corresponds to deprotonation of HPTS. Geometry optimizations show that the final experimental spectral shift is within $0.02 \mathrm{eV}$ of the shift computed with large proton solvent separation, indicating that the 78 ps experimental spectral shift is due to diffusion of the proton away from HPTS, rather than a single deprotonation event.

\section{Experimental Procedures}

The HPTS (8-hydroxypyrene-1,3,6-trisulfonate) used in the experiments was provided by Dr. Ernst Koller at Lambda Probes \& Diagnostics. Commercially available HPTS is contaminated by an unknown fluorescent impurity that causes problems in the analysis of the experimental data. The samples used here are free from contamination. MPTS (8-methoxypyrene-1,3,6-trisulfonate) 
was purchased from Sigma Aldrich. All chemicals were used as received. In the experiments, 1 mm path length cuvettes were filled with $\mathrm{H}_{2} \mathrm{O}$ or $\mathrm{D}_{2} \mathrm{O}$ solutions of $10^{-4} \mathrm{M}$ HPTS or MPTS for the fluorescence measurements.

Time resolved fluorescence decays were measured using a time-correlated single photon counting (TCSPC) setup described previously. ${ }^{30}$ Approximately 100 fs laser pulses centered at 730 nm were generated by a Ti:Sapphire oscillator (Spectra Physics MaiTai). An acousto-optic single pulse selector was used to select pulses from the $80 \mathrm{MHz}$ laser train at $5 \mathrm{MHz}$. The selected pulses were doubled in a barium borate crystal, yielding $365 \mathrm{~nm}$ light to excite HPTS and MPTS. The samples were excited and measured from the front surface in a near-normal geometry. Excitation occurred through a hole in a large lens, which was used to collect and collimate the emitted fluorescence. Another lens focuses the fluorescence on into the monochromator slit. The emission was detected at the magic angle polarization. The frequency of the fluorescence was resolved by the monochromator, and the single photons were detected by a multichannel plate (MCP) detector. The fluorescence decays were collected from $400 \mathrm{~nm}$ to $600 \mathrm{~nm}$ in $2 \mathrm{~nm}$ increments by stepping the monochromator. The wavelength scan was repeated multiple times. Entire fluorescence decays were obtained at each wavelength, and time dependent spectra were assembled from the time decays at each wavelength. The instrument response function (IRF) was collected using a weakly scattering suspension in water in the same cuvette used to measure the time dependent fluorescence.

\section{Experimental Results and Discussions}

Figure 1 displays time dependent fluorescence spectra of excited HPTS in $\mathrm{H}_{2} \mathrm{O}$ and $\mathrm{D}_{2} \mathrm{O}$ from 30 ps to $>25 \mathrm{~ns}$. The data were taken as described above. A decay curve was acquired at each wavelength (2 $\mathrm{nm}$ steps), and then the intensities at each wavelength at a particular time were

plotted. Figure 1A shows the data for HPTS in $\mathrm{H}_{2} \mathrm{O}$; Figure $1 \mathrm{~B}$ shows HPTS in $\mathrm{D}_{2} \mathrm{O}$; and Figure 
1C shows MPTS data in $\mathrm{H}_{2} \mathrm{O}$. The initial HPTS excited protonated state decays into the associated state in $3.5 \mathrm{ps},{ }^{13}$ which is faster than the 30ps (with deconvolution) time resolution of the TCSPC system. In Figures 1A and B, the states labeled "associated" and "deprotonated" are at higher/lower frequency, respectively. The curves are for the times given in Figure 1A. In both $\mathrm{H}_{2} \mathrm{O}$ and $\mathrm{D}_{2} \mathrm{O}$, the associated peak decays and the deprotonated peak grows in. The process is considerably slower in $\mathrm{D}_{2} \mathrm{O}$ than in $\mathrm{H}_{2} \mathrm{O}$. At very long time, the associated state maintains some amplitude. As discussed in the introduction, following deprotonation, proton (hydronium) diffusion repopulates the associated state to a small extent. The decay of the associated state into the deprotonated state is exponential followed by a low amplitude power law that arises from the diffusive repopulation of the associated state. ${ }^{12-13}$

Figure 1C shows the time dependent spectrum of MPTS. MPTS is identical to HPTS except that the HPTS hydroxyl is replaced by a methoxy. Therefore, it does not deprotonate in the excited state. The spectra were taken under identical conditions as the HPTS data. The lifetime decay of the amplitude was removed from the data so that it can be seen that the band is independent of time because there is no proton dissociation. The peak position is at higher frequency than the associated peak, and is more akin to the initial protonated state of the HPTS.

Figure 2 shows several of the HPTS spectra from Figure 1 with fits to the band shapes. At long time (>5 ns) the spectrum is essentially only the deprotonated band. The long time deprotonated band shape was fixed and only its amplitude was allowed to vary. The associated band shape and amplitude were allowed to vary, but its shape was held constant over time. In each panel, the black curve is the experimental spectrum. The red curve is the fit. It overlaps so well with the black curve that the black curve is substantially obscured. The blue and green curves are the band shapes of the associated and protonated states. The peak positions of the bands are: $\mathrm{H}_{2} \mathrm{O}$ 
associated, $446 \pm 2 \mathrm{~nm} ; \mathrm{D}_{2} \mathrm{O}$ associated, $444 \pm 2 \mathrm{~nm} ; \mathrm{H}_{2} \mathrm{O}$ deprotonated, $510 \pm 2 \mathrm{~nm}$; and $\mathrm{D}_{2} \mathrm{O}$ deprotonated, $512 \pm 2 \mathrm{~nm}$. These types of fits allow the areas of the bands to be obtained as a function of time. The total area, i. e., the sum of the areas of the two bands, decays with the fluorescence lifetime, $4.55 \mathrm{~ns}^{12}$

Figure 3 displays the decay of the associated state for HPTS in $\mathrm{H}_{2} \mathrm{O}(\mathrm{A})$ and in $\mathrm{D}_{2} \mathrm{O}$ (B). The data are the time dependent areas of the associated state divided by the total area of the total spectrum (associated plus deprotonated bands) at each time. Dividing by the total area removes the lifetime decay from the data. Therefore, the data represents only the decay of the associated state into the deprotonated state. The decay is an exponential at short time and a power law at long time. As the deprotonated state is formed, the protons diffuse away from the HPTS. A small fraction returns to HPTS to recreate the associated state, producing a small associated state population for times very long compared to the exponential decay of the associated state into the deprotonated state. ${ }^{23}$ The reformed associated state again decays into the deprotonated state, and the proton diffuses away. As time increases, protons diffuse out of the vicinity of HPTS, and fewer recombine. This process leads to the power law decay. ${ }^{22}$ At very long time, the power, $t^{-\alpha}$, will asymptotically approach $\alpha=1.5 .^{22-23}$ At short time, theory indicates that $\alpha=1.4$, which has been observed experimentally. ${ }^{12,21}$

The data in Figure 3 were fit in the following manner. The functional form starts as an exponential and then makes a smooth transition into the power law, which grows in as the exponential decays. The curve is not the sum of an exponential and a power law as recombination, which gives rise to the power law, cannot occur until the deprotonated state becomes populated. In this work, we are primarily interested in the short time dynamics where the exponential behavior dominates. This is in contrast to prior experiments where the power law was the focus and data 
were taken to much longer time. ${ }^{12,21,23}$ As the power exponent was found to be 1.4 from both experiment and theory, in the fits, $\alpha$ was fixed at 1.4 . The exponential decay constants are $78 \pm 2$ ps and $219 \pm 2$ ps in $\mathrm{H}_{2} \mathrm{O}$ and $\mathrm{D}_{2} \mathrm{O}$, respectively.

In the following sections, ab initio molecular dynamics simulations are applied to examine the HPTS proton transfer dynamics. The initial processes immediately following excitation, the nature of the associated state, and the final proton dissociation are explicated. Although the simulations agree with the observed time constants, the molecular picture of the structures involved in the "associated" and "deprotonated" states are found to be more complex than the simple picture inferred from experiments and described above.

\section{Ab Initio Molecular Dynamics Simulations Methodology}

In order to investigate this system, the computational work comprised several different steps, following our previous work. ${ }^{30}$ We first performed classical molecular dynamics simulations on the ground state and extracted equilibrated structures. These were then used to investigate the excited state surface using quantum mechanics/molecular mechanics (QM/MM) BornOppenheimer molecular dynamics (BOMD). From these trajectories, we extracted microsolvated clusters to compute stationary points using QM methods, and computed barriers along minimum energy paths connecting these structures using the improved dimer method, ${ }^{31}$ as described in the SI.

The HPTS force field parameters used in ground state equilibration were taken from previous work. ${ }^{30}$ In brief, the partial charges were obtained with RESP fitting, ${ }^{32}$ and most of the geometric parameters were taken from the general Amber force field (GAFF). ${ }^{33}$ The sulfate group S-O parameters for HPTS were taken from the literature. ${ }^{34}$ The water model used was TIP3P, with relevant ion parameters for $\mathrm{Na}^{+} .35$ The HPTS parameters used are provided in the SI.

Walker, et al. - Proton Transfer in HPTS - Page 9 
The classical empirical force field trajectory had a single HPTS molecule coordinated by $3 \mathrm{Na}^{+}$ions and solvated in a water box of 4277 waters $(50 \AA)$ using periodic boundary conditions. An initial structure of solvated HPTS was created with tleap. The structure was minimized, equilibrated with NPT at 1 atm/10 K for 40 ps, slowly heated to $300 \mathrm{~K}$ over $100 \mathrm{ps}$ in NVT, and then equilibrated for 40 ps ( $1 \mathrm{~atm} / 300 \mathrm{~K} / \mathrm{NPT})$. This was followed by $100 \mathrm{~ns}$ of production NPT simulation at $1 \mathrm{~atm}$ and $300 \mathrm{~K}$. These were run in duplicate, using pmemd.cuda from Amber. ${ }^{36-37}$ A Langevin thermostat with $5 \mathrm{ps}^{-1}$ friction parameter was used to maintain the temperature. ${ }^{38}$

Snapshots from the ground state dynamics were clustered according to the hydroxyl O-H bond distance and the distance from the $\mathrm{H}$ atom of the HPTS hydroxyl to the $\mathrm{O}$ atom of the closest water. Outlying waters were removed to create a sphere of $\sim 18 \AA$ (1000 waters), to be used for excited state QM/MM AIMD. Several QM regions were tested-4 waters were insufficient to see deprotonation in dynamics, while two solvation shells ( 100 waters) around the HPTS molecule was prohibitively expensive for the desired 3 ps run time. The final QM region chosen included two solvation shells around the hydroxyl moiety, spanning the distance between the $\mathrm{OH}$ and the closest HPTS sulfate which the $\mathrm{OH}$ pointed toward ( 30 waters). This was sufficient to see deprotonation within 3 ps or less of BOMD dynamics, and enough to converge the charge description of the HPTS hydroxyl moiety as described below.

All electronic structure calculations were performed with TeraChem, ${ }^{39-40}$ interfaced with OpenMM $\mathrm{M}^{41}$ to describe the $\mathrm{MM}$ region. For geometry optimizations, we used the $\omega \mathrm{PBE}$ method with range separation parameter $\omega=0.35$ and the $6-31 \mathrm{~g} * *$ basis set, as in previous work. ${ }^{30}$ The range separated functional avoids spurious low-lying charge transfer excited states (which would otherwise be ubiquitous because of the presence of the Na cations). Polarizable continuum implicit 
solvation $^{42-44}$ was also used for a set of geometry optimizations and some of the transition state optimizations. For the QM/MM BOMD, an additional d3 dispersion correction was added. ${ }^{45-46}$

QM/MM dynamics (NVE with a 1 fs time step) were performed on $\mathrm{S}_{1}$, with 5 initial conditions taken from the classical MD trajectory. An additional $6^{\text {th }}$ trajectory on $\mathrm{S}_{0}$ was run to ensure that no deprotonation occurs on the ground state within 4 ps. Each snapshot was cut into a sphere as described above and simulated using spherical boundary conditions. The classical trajectories were clustered based on the HPTS hydroxyl $\mathrm{O}-\mathrm{H}$ distance and the equatorial/axial disposition of the $\mathrm{OH}$ group (as discussed in previous work, the axial conformer has the $\mathrm{OH}$ pointing along the short axis of the HPTS molecule and the equatorial conformer has the $\mathrm{OH}$ pointing along the long axis of HPTS). ${ }^{30}$ The centroid snapshot for each cluster was extracted, and the radial distribution function (RDF) (water to the H atom of the HPTS hydroxyl group) was computed for each. Five snapshots from this pool were chosen to cover the range of O-H distance and $\mathrm{O}-\mathrm{H}$ orientation (axial vs. equatorial). Each snapshot was run for $10 \mathrm{fs}$ on $\mathrm{S}_{0}$ to minimize artifacts from changing the description from MM to QM/MM, and then run for 600 fs to 2.5 ps in the NVE ensemble on $S_{1}$. Trajectories were stopped a short time after the proton localized to within 1 water of the QM/MM boundary for at least $100 \mathrm{fs.} \mathrm{We} \mathrm{track} \mathrm{the} \mathrm{proton} \mathrm{through} \mathrm{the} \mathrm{wire} \mathrm{using} \mathrm{a}$ collective coordinate previously described by König et al. ${ }^{47}$ and originally suggested by Chakrabarti et al., ${ }^{48}$ as discussed in detail below.

\section{Ab Initio Microsolvated Geometry Optimizations}

The ground state and excited state dynamics calculations are accompanied by structural optimizations of microsolvated cluster geometries in gas phase and implicit solvent. These optimizations start with geometries taken from the snapshots from the ground state QM/MM-MD trajectories. We found that 6-8 explicit QM water molecules are needed in order to stabilize the 
deprotonated structures. When these snapshots are minimized on $\mathrm{S}_{0}$, the retained water molecules do not rearrange significantly. However, minimization on $S_{1}$ leads to a large-scale rearrangement of the waters to form a wire. This rearrangement is consistent for different starting structures. Therefore, the reported $S_{0}$ minimum is obtained from an optimization starting from one of these $S_{1}$ structures, minimizing ground/excited-state differences in water rearrangement of the microsolvated systems. Individual sodium atoms were positioned to coordinate with the HPTS sulfate groups prior to minimization. Further details for the transition state optimization can be found in the SI.

\section{Collective Coordinate Methods}

Collective coordinates were computed by determining the proportion of proton localization as a function of distance, computing the coordination number of each water in the wire based on those proportions, and relating it back to the overall length of the water wire as shown in the literature. ${ }^{47}$ To determine a coordination number, we first use the following switching function $f_{s w}(r)$ :

$$
f_{s w}(r)=\frac{1}{\left.1+e^{(r} 1.4 \AA\right) / 0.05 \AA}
$$

which drops off quickly for distances greater than $1.4 \AA$. The excess proton coordination number for each water molecule $\left(w^{O_{i}}\right)$ is then given by:

$$
w^{O_{i}}=\left(\sum_{j=1}^{N_{H}} f_{s w}\left(r_{O_{i} H_{j}}\right)\right) \quad N_{H}^{r e f}
$$

where $r_{O_{i} H_{j}}$ is the distance between the $i$ th oxygen atom and the $j$ th hydrogen atom, $N_{H}$ is the total number of hydrogen atoms in the system, and $N_{H}^{r e f}$ is the number of protons which are attached to the oxygen in its reference state ( 2 for water molecules and 0 for the HPTS OH group). With this 
definition, water molecules with no excess proton character have $w=0$ and an idealized hydronium has $w=1$. We compute the excess proton coordination number for each QM oxygen atom in the simulation after $1 \mathrm{ps}$ in order to determine which water molecules are involved in the water wire (these have $w>0$ ). Once the water molecules in the wire are known, we compute the collective coordinate $v$ as:

$$
v=\frac{{ }_{i=1}^{N_{O}} r_{z}^{O_{i}} w^{O_{i}}}{{ }_{i=1}^{N_{O}} w^{O_{i}}}
$$

where $r_{z}^{O_{i}}$ is the sum of the $\mathrm{O}-\mathrm{O}$ distances along the wire to the $i$ th oxygen atom. This gives the location of the excess proton as a function of distance along the wire, while taking into account the overall expansion and contraction of the wire that can occur in the simulation. It is important to note that this is a distance along the wire; if the wire is curved this distance will be longer than a straight line drawn between the location of the proton and the HPTS hydroxyl moiety.

\section{Simulation Results and Discussion}

\section{Dynamics}

We extracted five structures from the ground state dynamics to explore the first excited state surface $\left(\mathrm{S}_{1}\right)$ with BOMD. These five snapshots were chosen from cluster analysis as described above to cover variations in $\mathrm{O}-\mathrm{H}$ distance and equatorial/axial disposition of the hydroxyl group. A representative structure can be seen in Figure 4.

We investigated several sizes of the QM region to balance computational cost while retaining the ability to describe deprotonation and the initial stages of the proton shuttling. Single point energies were computed for a representative snapshot with progressively larger numbers of surrounding explicit QM water molecules. Figure S1 shows the evolution of the excitation energy and the Mulliken charges for the HPTS OH group as a function of increasing numbers of QM 
waters. We find that the $\mathrm{OH}$ charge stabilizes at around 17 waters, i.e., including all water molecules within $3 \AA$ of the $\mathrm{O}$ atom in the HPTS hydroxyl group (measured by O-O distance). The excitation energy is converged to better than $0.1 \mathrm{eV}$ after inclusion of 17 water molecules. We therefore used two solvation shells around the HPTS hydroxyl (30 QM water molecules) for subsequent dynamics calculations. Exploratory calculations revealed that including four QM water molecules surrounding the HPTS hydroxyl was insufficient to observe any deprotonation within 3 ps of simulation and also that the dynamics observed (up to $600 \mathrm{fs}$ ) when including 100 QM water molecules was very similar to that observed with $30 \mathrm{QM}$ water molecules. Figure 5 shows the QM region of a representative trajectory at varying timescales, indicating a consistent area of QM waters remaining throughout the trajectory and reorganization within the QM region, without intrusion of MM water molecules. RDFs were computed for each trajectory to confirm this, showing that MM waters never get closer than $3.8 \AA$ to the HPTS hydroxyl, most likely due to the comparatively short timescale of the simulations. (Figure S2).

In general, each dynamics trajectory follows a similar path (on varying timescales), depending on the specific initial snapshot selected. Upon excitation, the hydrogen bond between the closest water molecule and the HPTS hydroxyl group is formed and/or strengthened within $\sim 100 \mathrm{fs}$ (as indicated by shortening of the O-O distance). In cases where there is already a wellformed H-bond between HPTS and a water molecule on the ground state, the O-O distance decreases to $2.5 \AA$ or less. The next 200-2000 fs, depending on the specific trajectory, involve a rapid and large rearrangement of the QM water (Figure 5). Water molecules up to $6 \AA$ away from the hydroxyl group rearrange to form a water wire. This initial reorganization results in a small red shift in emission energy of less than $0.05 \mathrm{eV}$. Experiments observed an emission red shift of $0.2 \mathrm{eV}$, occurring with a 3 ps time constant. Our results indicate that this shift is not due to the 
rearrangement/tightening of hydrogen bonds in the network, which occurs much faster than the observed 3 ps time constant. Therefore, we do not attribute this to an "associated" state geometry.

The form of the resulting water wires depends on the equatorial/axial disposition of the HPTS hydroxyl group. When the $\mathrm{OH}$ group is equatorial, the wire extends from the $\mathrm{OH}$ group to the sulfate which is closest along the long axis of HPTS (typically involving six water molecules), and when the $\mathrm{OH}$ group is axial, it extends from the $\mathrm{OH}$ group to the sulfate which is closest along the short axis (typically involving three water molecules). Water wire formation and organization from nearby charged functional groups has been observed in other simulations of excited state dyes and for proton transfer in organized environments, such as proteins, as discussed in the Introduction. Unlike the more randomized formation of these networks in bulk water, the negatively charged sulfate group appears to favor a consistent formation of this water wire across different trajectories. Once this wire forms, HPTS deprotonates and the proton is briefly shared within the network (Figure 6A/6C) before localizing on a specific water. A representative structure is shown in Figure 5B, where the proton localizes on a water molecule midway along the water wire (labeled "Water 2" in Figure 6).

This general pattern is followed for all the trajectories tested that include deprotonation events (four out of five of the initial conditions led to deprotonation on the 3 ps time scale of the simulation). The binned emission energies over time for all five trajectories are given in Figure 6D, showing a Stokes shift of almost $0.2 \mathrm{eV}$ by 2500 fs. We compute a collective coordinate as described above to relate these wavelength shifts to the location of the proton within the water wire, and further to its relative distance from the anionic oxygen of deprotonated HPTS (Figure 7). For the same trajectory shown in Figure 6, we find that the proton has rest phases where it oscillates between the HPTS and the first water in the wire, and burst phases where the proton is highly 
delocalized and then localizes on one of the water molecules in the wire. This is quite similar to previous theoretical findings for ground state excess proton dynamics in bulk water. ${ }^{7}$ In Figure 7A, the presence of a tightly bound third hydrogen on the relevant water is shown in yellow. Regions where two or more waters have considerable excess proton character (e.g. around 975 fs in Figure 7A) indicate an overall contraction of the water wire and strong sharing between the waters. Figure 7B shows the excess proton distance along the water wire over time, with an inset of the water wire geometries during the burst phase. This data suggests that if a traditional Zundel cation is involved, its lifetime is very short.

Another initial condition snapshot has preorganized waters and deprotonates within $100 \mathrm{fs}$. This trajectory was run both with 30 and 100 QM water molecules, yielding qualitatively similar results, as mentioned above. Figure 8 shows the trajectory with 100 QM waters. Interestingly, despite the much shorter timescale, Figure $8 \mathrm{~A}$ is quite similar in character to the burst phase in Figure 7A (compare the 600 fs shown in Figure 8A with the last 600 fs shown in Figure 7A). Such similarities are also observed when comparing Figures 8B and 8C to Figures 6A and 6B. This suggests that much of the variation in the relative timescales of deprotonation is due to latency in the water reorganization to form a water wire. Corroborating this is a particular trajectory (one out of the five that were modeled) that shows no deprotonation events, and also has little to no hydrogen bonding character that would assist with water wire formation (see SI for details).

The excited state natural orbitals for HPTS remain essentially unchanged over time and after deprotonation occurs (Figure 9), and do not involve surrounding water molecules to any significant degree at any stage. The excitation energies also do not drop below $2.7 \mathrm{eV}$ in any trajectory. This indicates that the excited state character is confined to the chromophore, suggesting that our observations may be generalizable to ground state proton transfer.

Walker, et al. - Proton Transfer in HPTS - Page 16 
Taken together, this is strong evidence that the initial shift in emission wavelength over the first 3 ps after photoexcitation does not arise from transition to an associated state where the proton is shared between the HPTS OH group and the nearest water molecule. Instead, it corresponds to localization of the excess proton on a water molecule located in the water wire, but not directly $\mathrm{H}$ bonded to the HPTS OH group.

\section{Optimizations}

To examine the origin of the process observed experimentally with a 78 ps time constant, we turned to microsolvated geometry optimizations, since the timescale was too long to directly observe with QMMM BOMD. Geometry optimizations were performed on single HPTS molecules neutralized with sodium ions and with 6-8 waters in a microsolvated cluster. These optimizations, similar to the dynamics trajectories, demonstrate an interesting dependence of emission wavelength on distance the proton has traveled from the HPTS oxygen (Figure 10A). If the proton has localized $9.1 \AA$ (about 3.5 waters) away, the emission wavelength redshifts by 0.30 $\mathrm{eV}$. This is very similar to the redshift observed experimentally on the 3 ps timescale $(0.28 \mathrm{eV})$. If the proton is moved one water further, the emission wavelength redshifts by $0.47 \mathrm{eV}$. Finally, if the proton is removed to an infinite distance away, the emission wavelength redshifts by $0.54 \mathrm{eV}$. This supports the assignment of the deprotonated state (experimental emission redshift of $0.45 \mathrm{eV}$ ) to proton diffusion away from the initial water wire.

We can compare these shifts to those obtained from the averaged dynamics trajectories and their collective coordinates for proton transfer, as discussed above (Figure 10B). The dynamics show strong qualitative similarity to the microsolvated optimizations, showing a redshift of $\sim 0.25$ $\mathrm{eV}$ when the proton is localized $7 \AA$ away. Figure 10B shows a very nearly linear relationship between emission energy and proton distance, and therefore provides a "ruler" with which one can determine approximately how far the proton has traveled by its emission wavelength.

Walker, et al. - Proton Transfer in HPTS - Page 17 
We investigated proton transfer between HPTS and its neighboring water with the most minimal possible rearrangement of waters in order to separate the question of whether the deprotonation process is barrierless from the mechanism of water reorganization. To this end, we optimized the corresponding transition state on $S_{1}$. We included a water cluster consisting of eight $\mathrm{H}_{2} \mathrm{O}$ molecules and surrounding implicit solvent, as described in the SI. The potential energy along the corresponding intrinsic reaction coordinate (IRC) is shown in Figure 11. The reaction has a small potential energy barrier of $2.2 \mathrm{kcal} / \mathrm{mol}$. Therefore, we can qualitatively confirm that the timescale for the initial deprotonation is far below the 78 ps timescale previously assigned to the deprotonated state.

Figure 11 furthermore shows that the vertical excitation energy shifts by $-0.1 \mathrm{eV}$ during the minimal deprotonation event. This supports the conclusion from the dynamics, that the shift for the "associated" structure is actually an initial short-range deprotonation event. It also implies that, rather than a completely stepwise mechanism where the proton hops one water at a time, it is more likely that the first step involves a proton hopping 2-3 waters away in a concerted motion over the course of 100-200 fs. This is further supported by the experimental redshift of $0.28 \mathrm{eV}$ after 3 ps as compared to the theoretical redshift from dynamics and optimizations $(0.15 \mathrm{eV} / 0.30$ $\mathrm{eV}$ shift for proton moving two/three waters away from HPTS along the wire).

\section{Concluding Remarks}

In this work, we presented the first $\mathrm{QM} / \mathrm{MM}$ simulations of photoexcitation dynamics in the solvated photoacid HPTS including sufficient QM water molecules to capture the proton transfer events. The strongly negative sulfate groups provide anchoring points for the formation of short, tight water wires on the excited state potential energy surface. The deprotonation of HPTS is gated by the formation of such a water wire extending from the HPTS OH group to a nearby 
sulfate. Previous theoretical work on protein systems has indicated the organization of water close to protons and protein residues to have a strong impact on the relative speed of the proton shuttling, with sub-mechanisms associated with faster and slower proton shuttles depending on the organization of solvent around the deprotonation site. ${ }^{49-52} \mathrm{We}$ have similar findings, with "preorganized" water leading to deprotonation within hundreds of femtoseconds, and less organized water leading to deprotonation on a 3 ps timescale. Interestingly, the $\mathrm{SO}_{3}{ }^{-}$moieties on HPTS appear to perform a similar function to negatively charged protein residues close to deprotonation sites, assisting the water to form wires in a relatively predictable fashion. Simulations of small molecule dyes, similar to HPTS, also show that the speed of proton shuttling depends on water organization at the moment of excitation. ${ }^{53}$ Furthermore, these same authors find that long water wires containing more than four water molecules are not very stable, and that concerted proton hopping mechanisms are usually limited to this range. ${ }^{53}$ Our results align well with this previous work.

We find a strong correlation between the emission wavelength and the distance between the HPTS oxygen atom and the excess proton in the water wire. This nearly linear relationship could be used to track proton motion in the wire with the measured emission wavelength. That said, to be conclusive one would need to test more initial conditions than we have in this work.

Our work provides a clear molecular picture of the intermediates involved in the excited state proton transfer of aqueous HPTS. The "intermediate" time constant of 2-3 ps observed in numerous experiments, ${ }^{13,18-19}$ previously assigned to a "shared" proton between HPTS and a coordinating water molecule, has been revealed to instead correspond to deprotonation of the HPTS hydroxyl group and rattling of the excess proton within a water wire. We also showed that the longer time constant of $\sim 80 \mathrm{ps}$ corresponds to diffusion of the excess proton away from this 
water wire. Previous interpretations of this process assumed proton diffusion from the water molecule coordinating the HPTS hydroxyl group.

\section{Supporting Information}

The supporting information is available free of charge at XX.

Details about $\mathrm{QM} / \mathrm{MM}$ region selection, collective coordinate chosen for $\mathrm{QM} / \mathrm{MM}$ dynamics, and transition state optimization methodology (PDF).

Optimized geometries for critical point structures and force field parameters for HPTS (ZIP).

\section{Acknowledgments}

This work was supported in part (A. R. W., J. M., and T. J. M.) by the U. S. Department of Energy, Office of Science, Office of Advanced Scientific Computing Research, Scientific Discovery through Advanced Computing (SciDAC) program and the Chemical Sciences, Geosciences, and Biosciences Division of the Office of Basic Energy Sciences, Office of Science, U. S. Department of Energy, AMOS program and also (B. W. and M. D. F.) by the National Science Foundation, Division of Chemistry, Award Number 1954392. We thank Dr. Ernst Koller, Lambda Probes \& Diagnostics, for providing us with the ultrapure HPTS used in the experiments. 


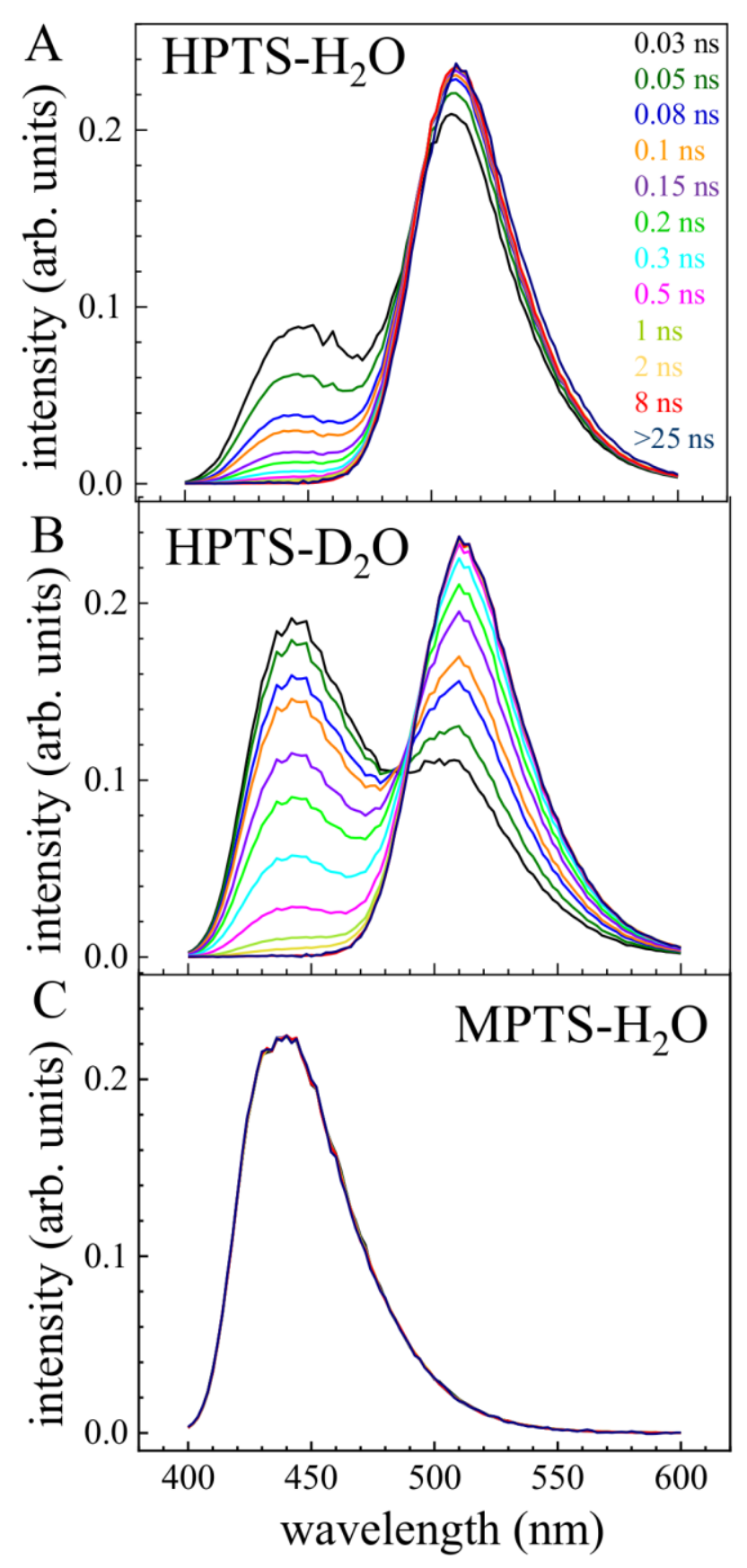

Figure 1. A. Data for HPTS in $\mathrm{H}_{2} \mathrm{O}$ from $30 \mathrm{ps}$ to $25 \mathrm{~ns}$. The high frequency band forms in $\sim 3.5$ ps due to the initial step in deprotonation. It then decays and the low frequency band grows in. The low frequency band is associated with complete deprotonation. It then decays with the excited state lifetime. The life decay has been eliminated from these plots. B. The same type of data as A but in $\mathrm{D}_{2} \mathrm{O}$. The decay of the high frequency band and the growth of the low frequency band are substantially slower in $\mathrm{D}_{2} \mathrm{O}$. C. MPTS data in $\mathrm{H}_{2} \mathrm{O}$. MPTS is identical to HPTS except that the HPTS hydroxyl is replaced by a methoxy. It does not deprotonate. Therefore, with the lifetime decay removed from the data, the spectrum is time independent. The times at which each spectrum was taken, shown in A, applies to all three figures. 


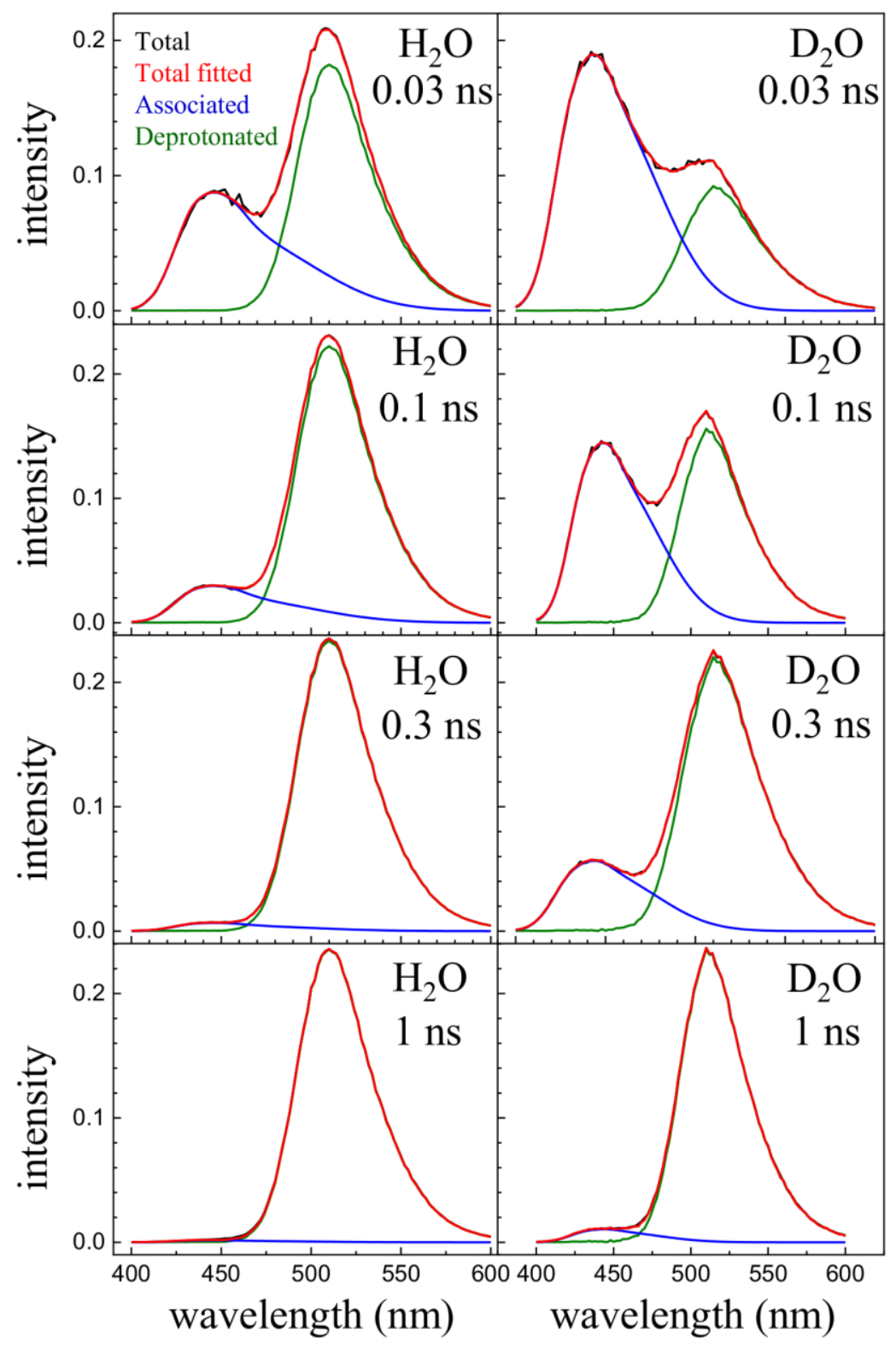

Figure 2. Several of the spectra from Figure 1 with fits to the band shapes in $\mathrm{H}_{2} \mathrm{O}$ and $\mathrm{D}_{2} \mathrm{O}$. At long time, $>5 \mathrm{~ns}$, the spectrum is essentially only the deprotonated band. The decay of the high frequency band into the low frequency fully deprotonated band is slower in $\mathrm{D}_{2} \mathrm{O}$ than in $\mathrm{H}_{2} \mathrm{O}$. 


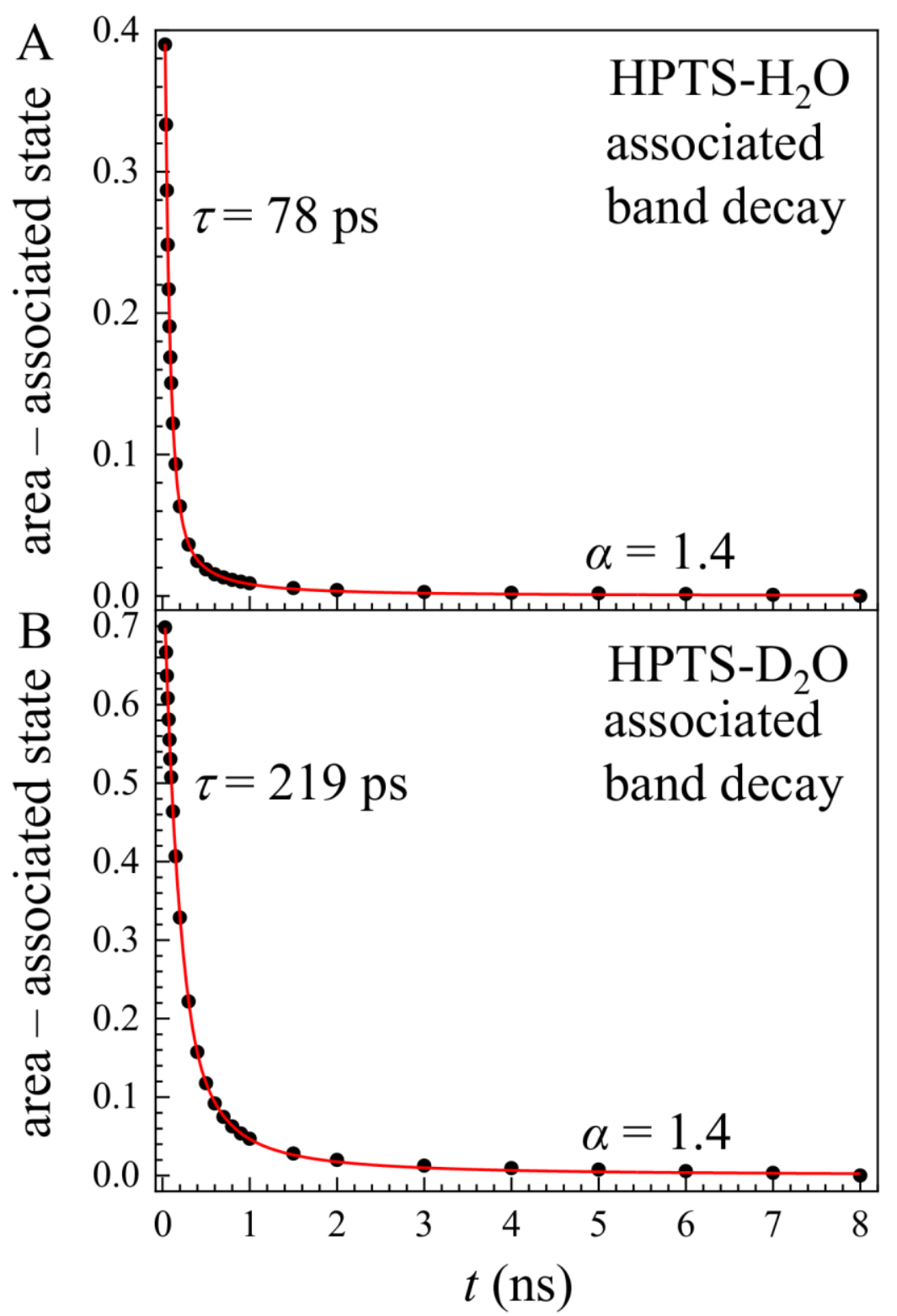

Figure 3. Decay of the high frequency band in figures 1 and 2 for HPTS in $\mathrm{H}_{2} \mathrm{O}(\mathrm{A})$ and in $\mathrm{D}_{2} \mathrm{O}$ (B). The data are the time dependent areas of the associated state divided by the areas of the total spectrum (associated plus deprotonated bands) at each time, which eliminates the lifetime decay from the data. The data decay exponentially at short time with decay constant $\tau$ and become a low amplitude power law, $t^{-\alpha}$, at long time. The power law arises from a small amount recombination following deprotonation and diffusion. 


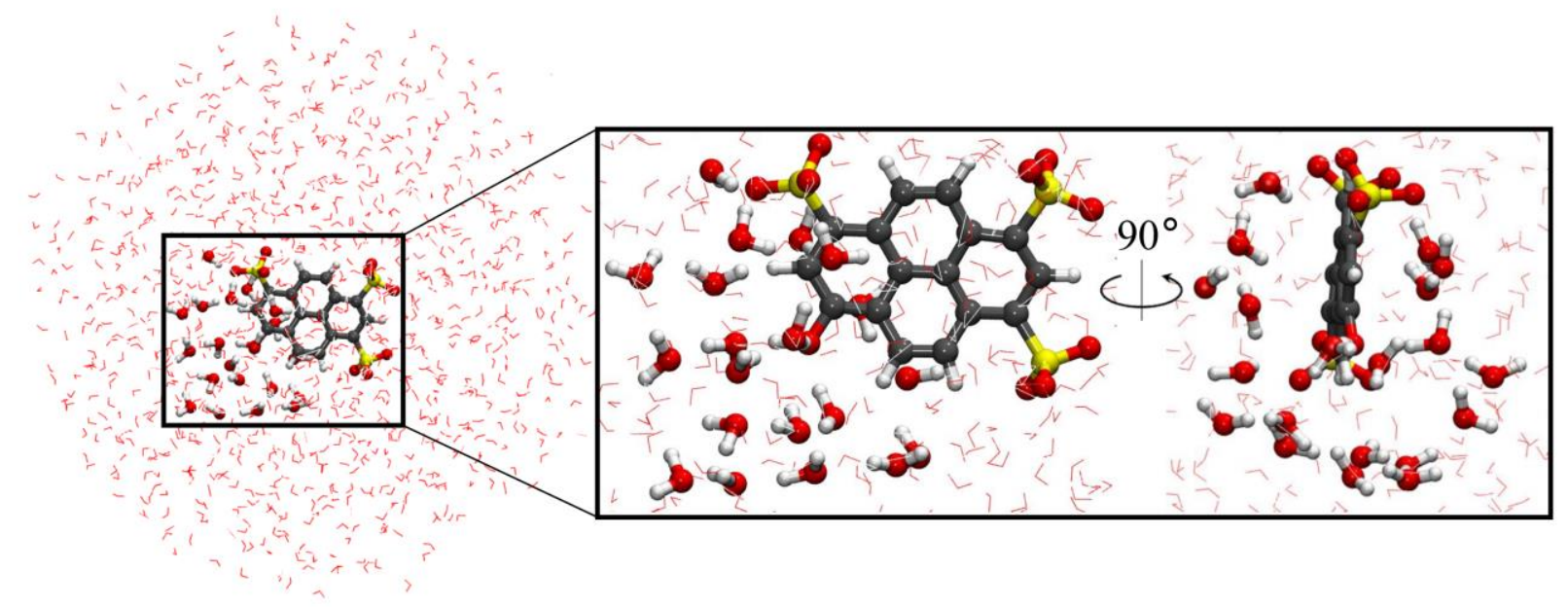

Figure 4. View of representative QMMM system (MM region in line representation and QM region in CPK representation). Zoom shows top and side close up views of the QM region. 


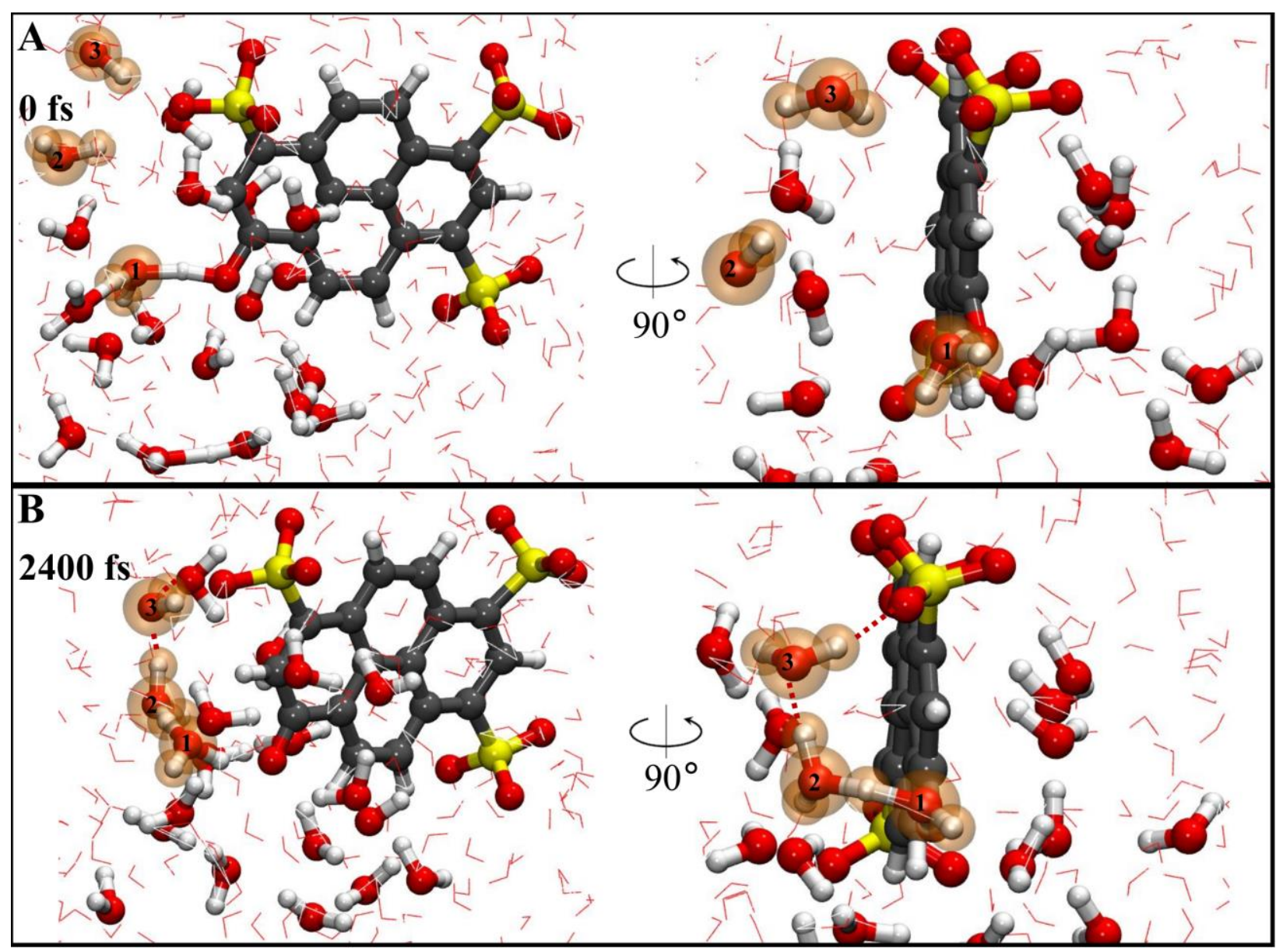

Figure 5. First (A) and last (B) structures from $2400 \mathrm{fs}$ trajectory, with waters contributing to the water wire coordinate labeled relative to their proximity to the HPTS hydroxyl group. The water molecules in the wire are identified (and numbered) by inspection at $2400 \mathrm{fs}$. Comparison of the 0 fs and 2400 fs frames shows that water molecules are "recruited" to form the water wire during the dynamics. CPK representation is used for the QM region and the MM region is depicted in line representation. 
A

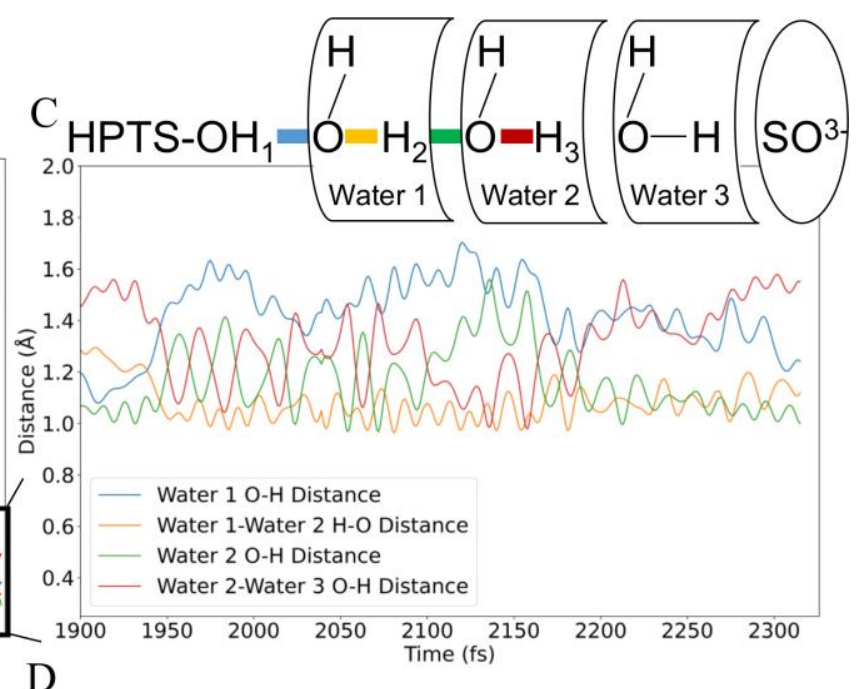

$\mathrm{B}$
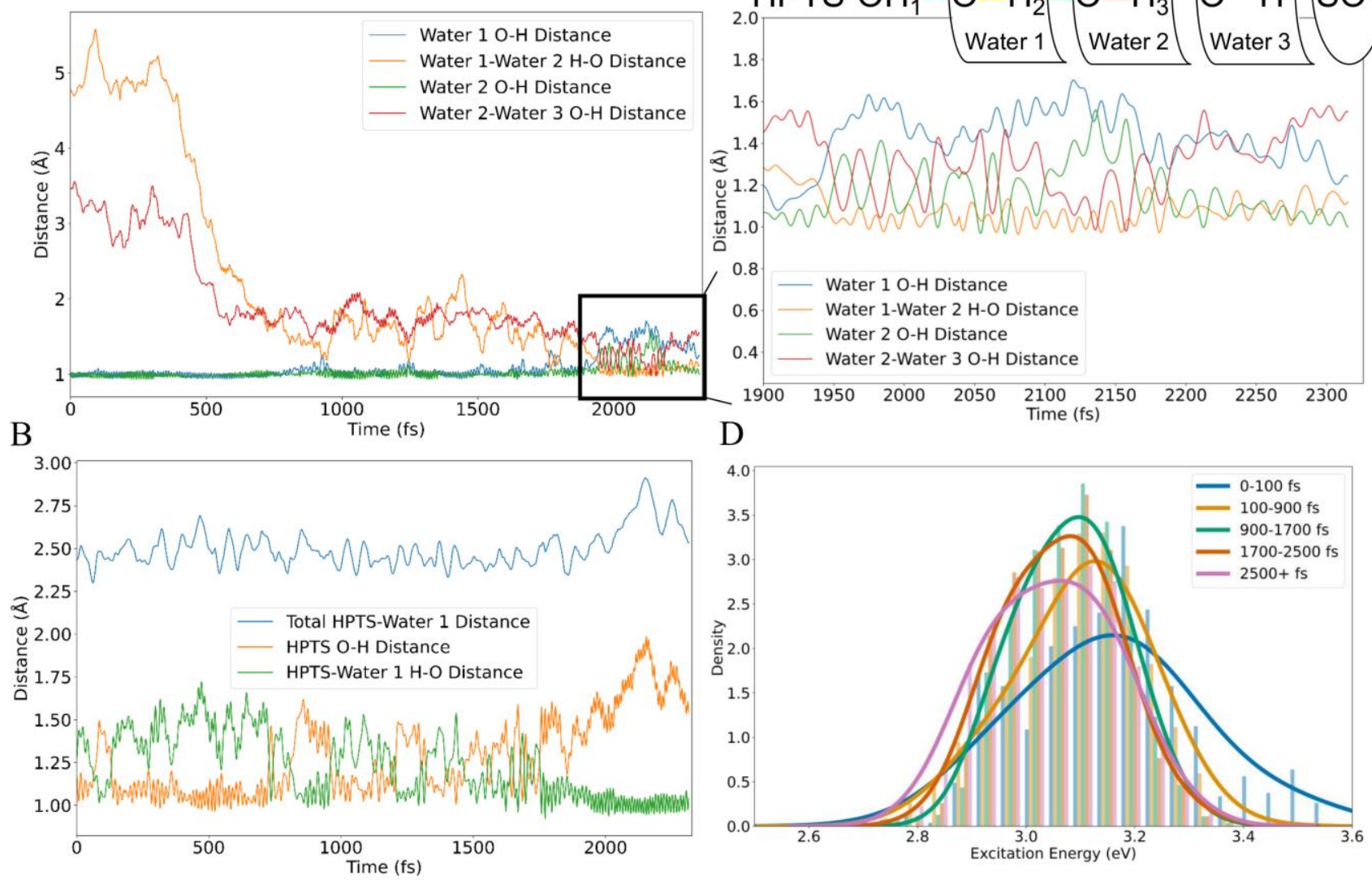

Figure 6. A. All O-H distances for the three waters shown in the water wire for a representative trajectory. B. Total distance between HPTS $\mathrm{O}$ and Water 1 (closest water) and the $\mathrm{O}-\mathrm{H}$ and $\mathrm{H}-$ $\mathrm{O}$ distances between the HPTS hydroxyl group and water. C. Zoom of the region of time where the proton is highly delocalized in the water wire, with a schematic of the relevant $\mathrm{O}-\mathrm{H}$ distances and wire organization with the same color legend as the graph D. Histogram of emission energies for all trajectories, binned by time window. 

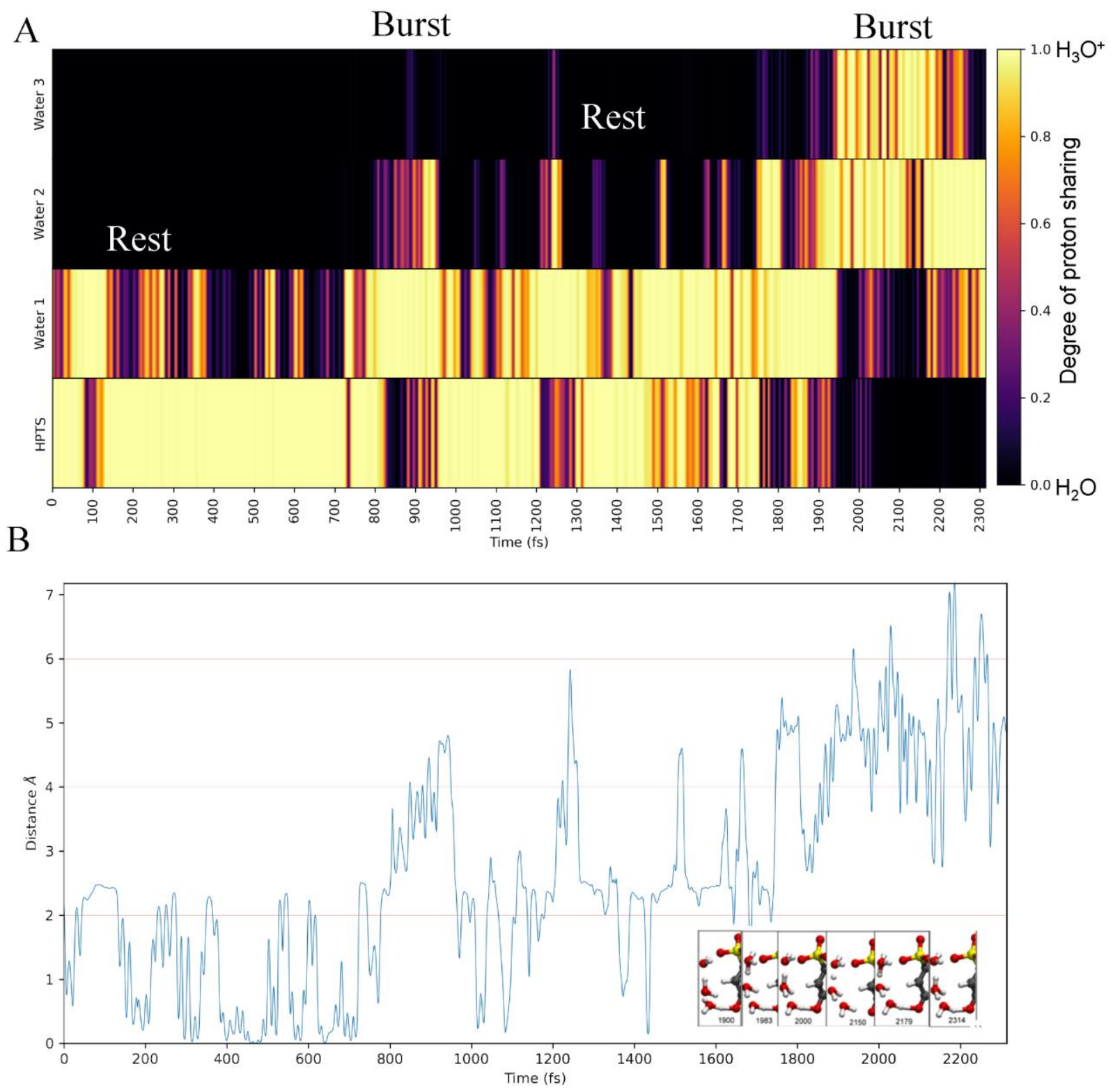

Figure 7. Burst and rest phases of proton sharing for the representative trajectory in Figure 6. A. Bar plot of each timestep for each water in the wire, colored by degree of proton sharing (Eq. 2) from 0 (black) to 1 (yellow). Black corresponds to a normal water, with no proton sharing character, while yellow corresponds to protonated HPTS or a water molecule with strong hydronium character. Areas of overlapping hydronium character indicate a combination of strong sharing between waters and an overall contraction of the water wire. B. Collective coordinate (Eq. 3) over time as a function of distance along the water wire in angstrom. 

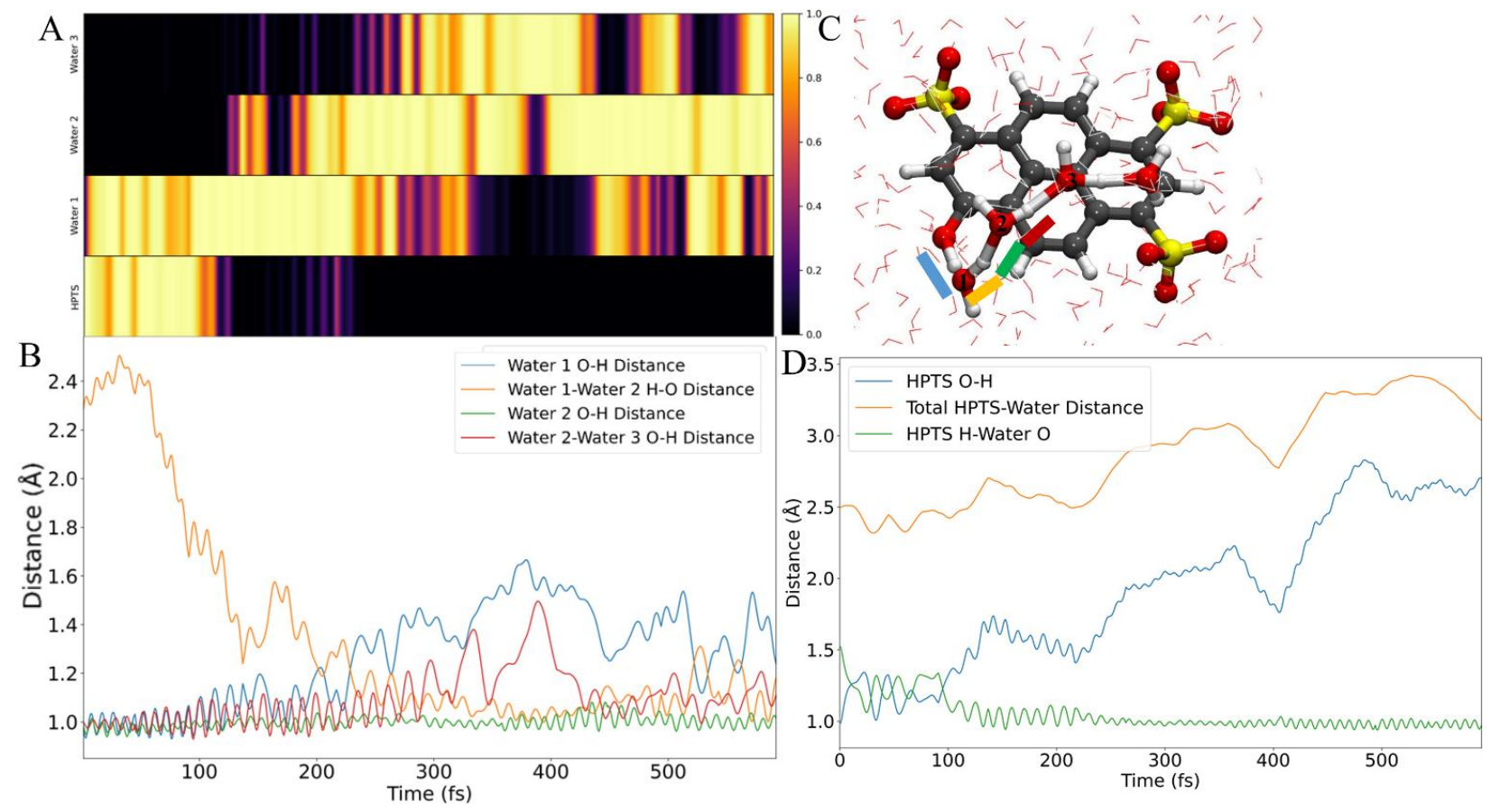

Figure 8. Composed plot of representative short trajectory with 100 waters in the QM region, showing almost instantaneous deprotonation. A. Bar plot of each timestep for each water in the wire, colored by degree of proton sharing as in Figure 7. B. Water O-H distances over the course of the trajectory. C. Total HPTS-Water distance (O-O distance from HPTS hydroxyl group to the nearest water molecule) and $\mathrm{O}-\mathrm{H}$ distances, showing rapid deprotonation in $100 \mathrm{fs}$ and the movement of the water wire away from the HPTS anion. D. Schematic of water organization after $100 \mathrm{fs}$, showing a preformed wire that promotes rapid deprotonation. 

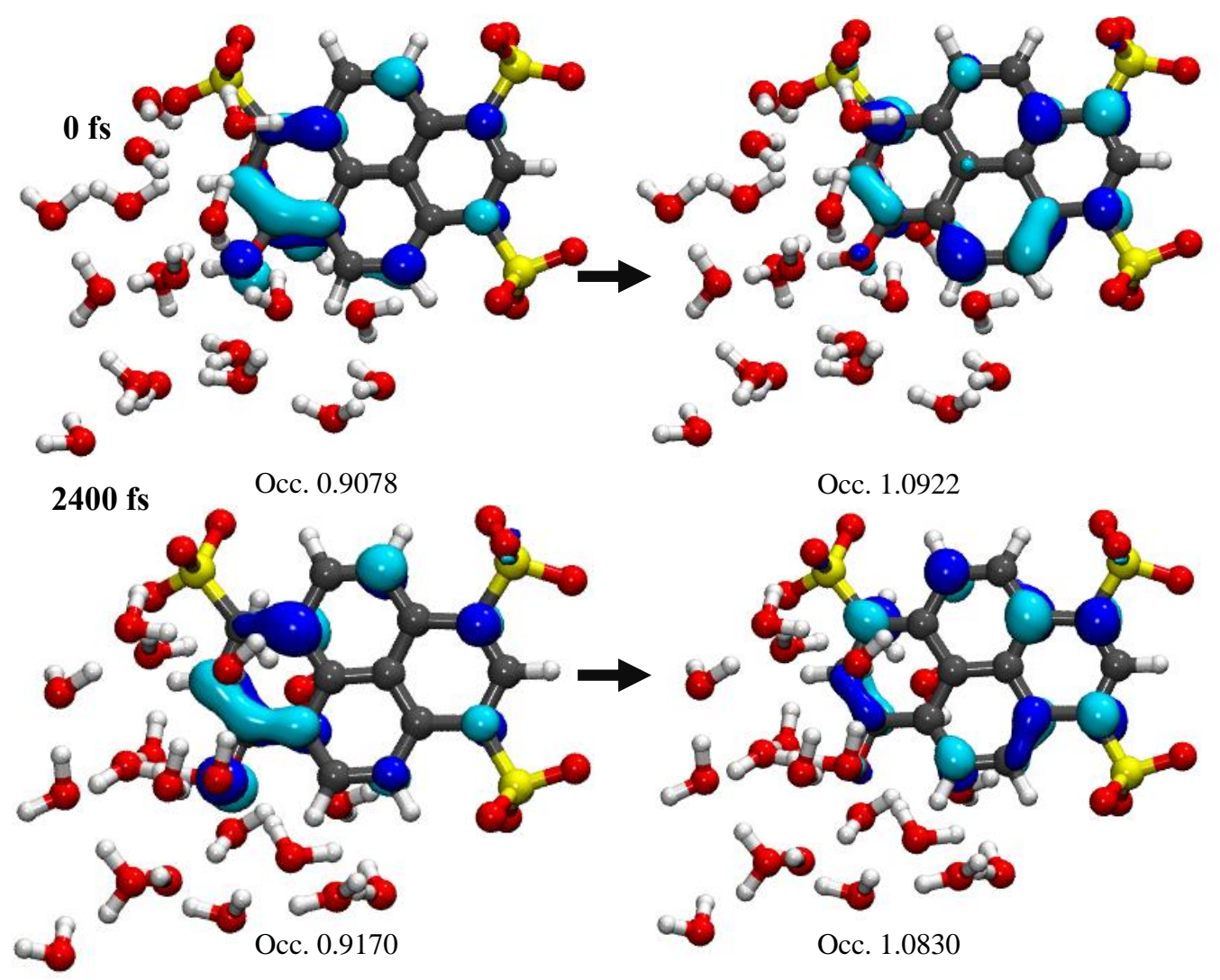

Figure 9. Transition natural orbitals for the $S_{1}$ transition at the beginning (top) and end (bottom) of dynamics. Throughout the dynamics, the excited state wavefunction is localized on HPTS and there is no significant involvement (i.e. charge transfer) from surrounding QM water molecules. 

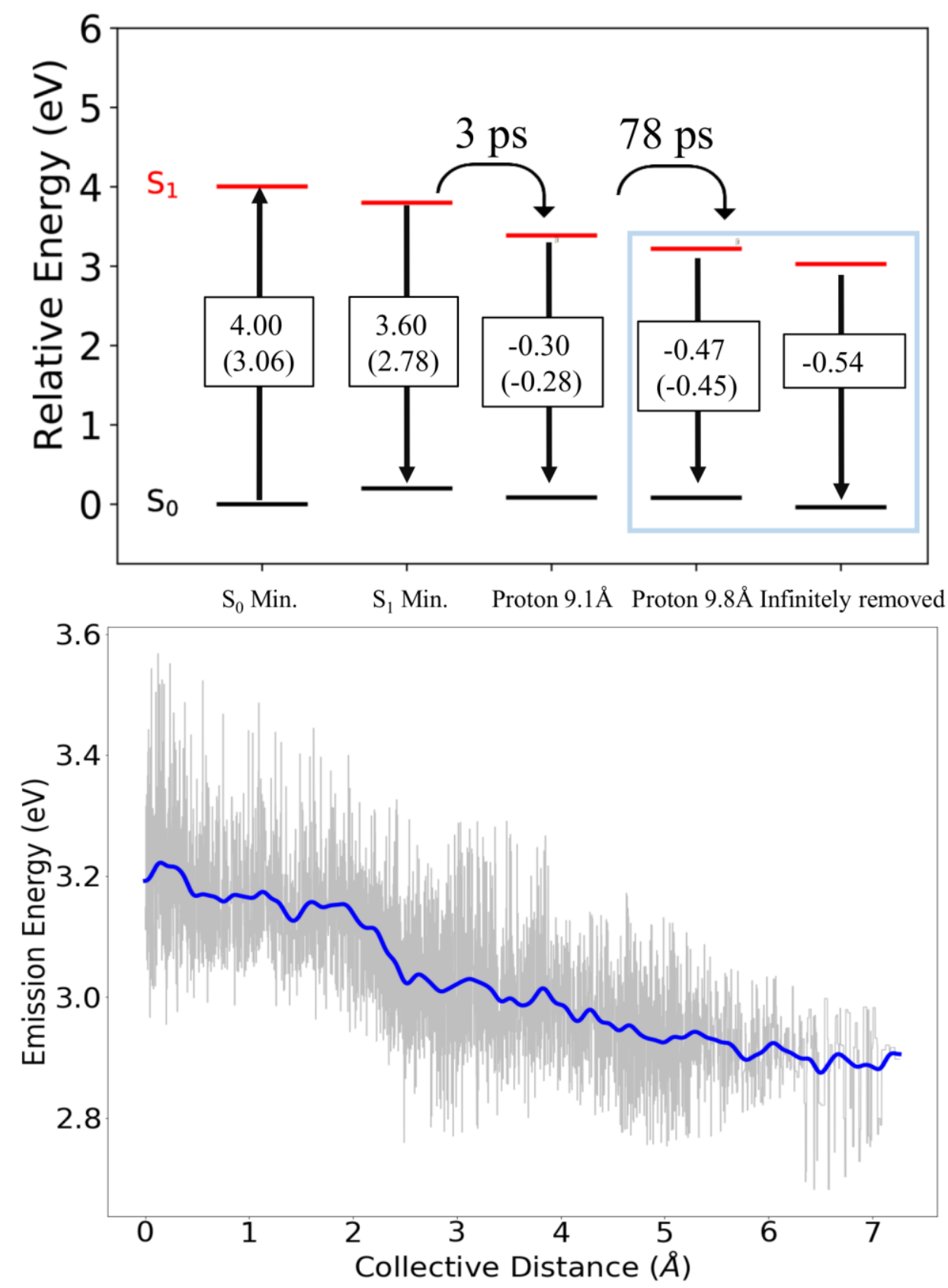

Figure 10. The top panel shows relative energies and $S_{1} / S_{0}$ energy gaps for various optimized geometries of microsolvated HPTS. These geometries correspond to the $S_{0}$ and $S_{1}$ minima for protonated HPTS and also $\mathrm{S}_{1}$ minima of deprotonated HPTS with the excess proton located at varying distances along the water wire. Emission energies are shown for the $S_{0}$ and $S_{1}$ minima of protonated HPTS. Relative emission shifts are shown for the three $S_{1}$ minima corresponding to deprotonated HPTS (experimental values in parentheses). Experimental timescales are marked above the relevant shifts. The bottom panel is computed from QM/MM dynamics with $~ 30$ waters in each QM region, and shows a collective coordinate (Eq. 3) vs. the excitation energy. This is combined data from all computed excited state trajectories. The final relative shift is approximately $-0.25 \mathrm{eV}$ for an excess proton located $7 \AA$ away from the HPTS oxygen (along the wire). 


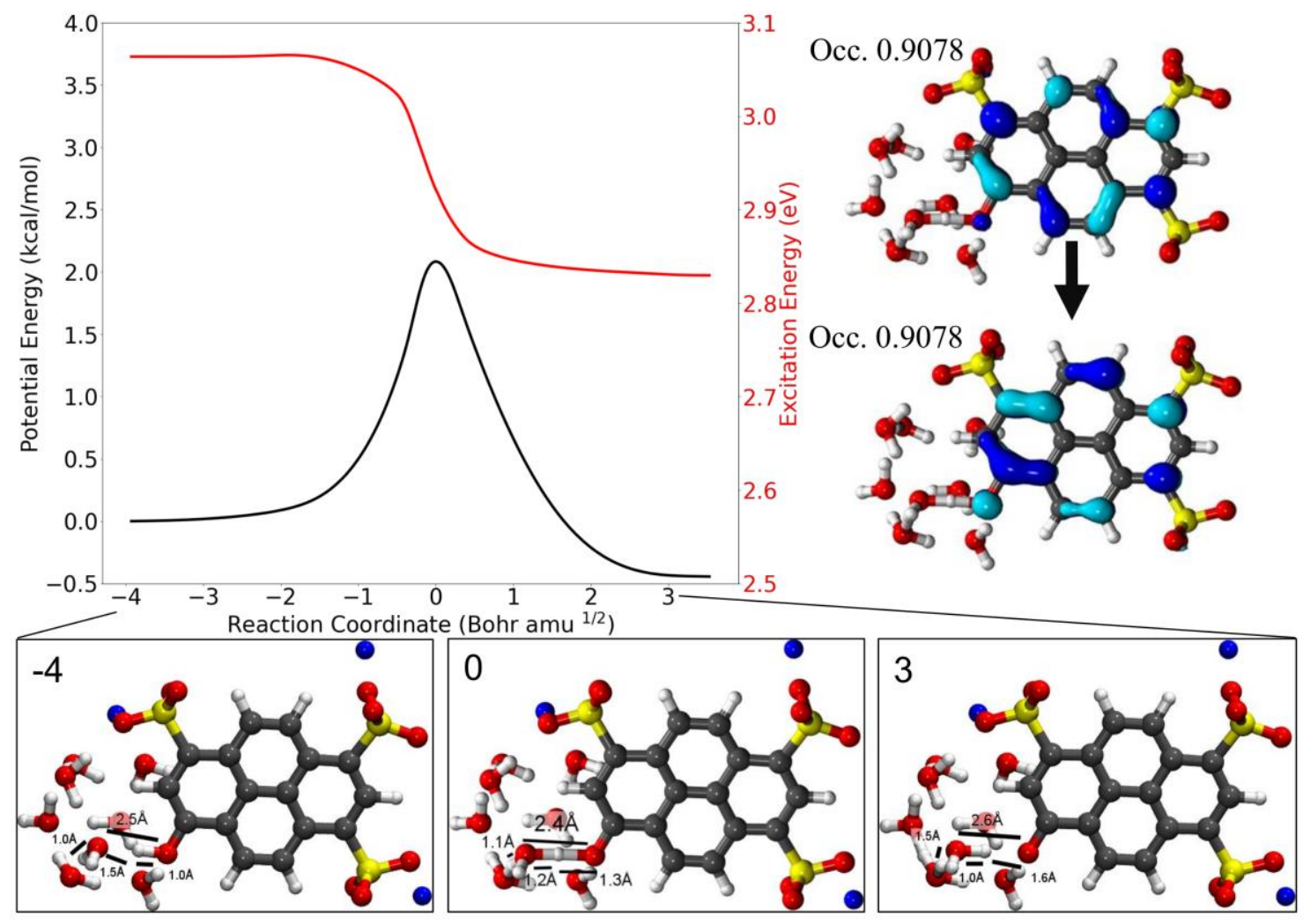

Figure 11. Potential energy on $S_{1}$ and vertical excitation energies along the intrinsic reaction coordinate corresponding to the deprotonation of HPTS. Here, $8 \mathrm{H}_{2} \mathrm{O}$ molecules and PCM have been used in combination with TD-DFT (omega=0.35-PBE/6-31G**). The shift corresponds to 4.1 $\AA$ in the collective coordinate. Structures along the path are shown below, with the transition natural orbitals associated with the transition state geometry shown on the right. 


\section{References}

1. Matheu, R.; Ertem, M. Z.; Benet-Buchholz, J.; Coronado, E.; Batista, V. S.; Sala, X.; Llobet, A., Intramolecular Proton Transfer Boosts Water Oxidation Catalyzed by a Ru Complex. $J$. Amer. Chem. Soc. 2015, 137, 10786.

2. Douhal, A.; Kim, S. K.; Zewail, A. H., Femtosecond molecular dynamics of tautomerization in model base pairs. Nature 1995, 378, 260.

3. Pedersen, B. P.; Buch-Pedersen, M. J.; Morth, J. P.; Palmgren, M. G.; Nissen, P., Crystal structure of the plasma membrane proton pump. Nature 2007, 450, 1111.

4. Yuan, R.; Napoli, J. A.; Yan, C.; Marsalek, O.; Markland, T. E.; Fayer, M. D., Tracking Aqueous Proton Transfer by Two-Dimensional Infrared Spectroscopy and ab Initio Molecular Dynamics Simulations. ACS Cent. Sci. 2019, 5, 1269.

5. Sofronov, O. O.; Bakker, H. J., Slow Proton Transfer in Nanoconfined Water. ACS Cent Sci 2020, $6,1150$.

6. Bekçioğlu, G.; Allolio, C.; Sebastiani, D., Water Wires in Aqueous Solutions from FirstPrinciples Calculations. J. Phys. Chem. B 2015, 119, 4053.

7. Hassanali, A.; Giberti, F.; Cuny, J.; Kühne, T. D.; Parrinello, M., Proton transfer through the water gossamer. Proc. Natl. Acad. Sci. 2013, 110, 13723.

8. Knight, C.; Voth, G. A., The Curious Case of the Hydrated Proton. Acc. Chem. Res. 2012, 45, 101.

9. Tolbert, L. M.; Solntsev, K. M., Excited-State Proton Transfer: From Constrained Systems to “Super” Photoacids to Superfast Proton Transfer. Acc. Chem. Res. 2002, 35, 19.

10. Agmon, N., Elementary Steps in Excited-State Proton Transfer. J. Phys. Chem. A 2005, 109, 13.

11. Rini, M.; Magnes, B.-Z.; Pines, E.; Nibbering, E. T. J., Real-Time Observation of Bimodal Proton Transfer in Acid-Base Pairs in Water. Science 2003, 301, 349.

12. Simkovitch, R.; Pines, D.; Agmon, N.; Pines, E.; Huppert, D., Reversible Excited-State Proton Geminate Recombination: Revisited. J. Phys. Chem. B 2016, 120, 12615.

13. Spry, D. B.; Goun, A.; Fayer, M. D., Deprotonation Dynamics and Stokes Shift of Pyranine (HPTS). J. Phys. Chem. A 2007, 111, 230.

14. Leiderman, P.; Genosar, L.; Huppert, D., Excited-State Proton Transfer: Indication of Three Steps in the Dissociation and Recombination Process. J. Phys. Chem. A 2005, 109, 5965.

Walker, et al. - Proton Transfer in HPTS - Page 32 
15. Mohammed, O. F.; Pines, D.; Dreyer, J.; Pines, E.; Nibbering, E. T. J., Sequential Proton Transfer Through Water Bridges in Acid-Base Reactions. Science 2005, 310, 83.

16. Siwick, B. J.; Cox, M. J.; Bakker, H. J., Long-range proton transfer in aqueous Acid-base reactions. J. Phys. Chem. B 2008, 112, 378.

17. Han, F.; Liu, W.; Fang, C., Excited-state proton transfer of photoexcited pyranine in water observed by femtosecond stimulated Raman spectroscopy. Chem. Phys. 2013, 422, 204.

18. Prayer, C.; Gustavsson, T.; Tran-Thi, T. H., What's New in the Proton Transfer Reaction from Pyranine to Water? A Femtosecond Study of the Proton Transfer Dynamics. AIP Conf. Proc. 1996, 364, 333.

19. Tran-Thi, T. H.; Gustavsson, T.; Prayer, C.; Pommeret, S.; Hynes, J. T., Primary ultrafast events preceding the photoinduced proton transfer from pyranine to water. Chem. Phys. Lett. 2000, 329, 421.

20. Sedgwick, M.; Cole, R. L.; Rithner, C. D.; Crans, D. C.; Levinger, N. E., Correlating proton transfer dynamics to probe location in confined environments. J Am Chem Soc 2012, 134, 11904. 21. Thomaz, J. E.; Lawler, C. M.; Fayer, M. D., Proton Transfer in Perfluorosulfonic Acid Fuel Cell Membranes with Differing Pendant Chains and Equivalent Weights. J. Phys. Chem. B 2017, $121,4544$.

22. Agmon, N.; Szabo, A., Theory of Reversible Diffusion-Influenced Reactions. J. Chem. Phys. 1990, 92, 5270 .

23. Agmon, N.; Pines, E.; Huppert, D., Geminate Recombination in Proton Transfer Reactions. J. Chem. Phys. 1988, 88, 5631.

24. Asbury, J. B.; Steinel, T.; Stromberg, C.; Corcelli, S. A.; Lawrence, C. P.; Skinner, J. L.; Fayer, M. D., Water Dynamics: Vibrational Echo Correlation Spectroscopy and Comparison to Molecular Dynamics Simulations. J. Phys. Chem. A 2004, 108, 1107.

25. Raucci, U.; Chiariello, M. G.; Rega, N., Modeling Excited-State Proton Transfer to Solvent: A Dynamics Study of a Super Photoacid with a Hybrid Implicit/Explicit Solvent Model. J. Chem. Theory Comput. 2020, 16, 7033.

26. Pines, E.; Huppert, D.; Agmon, N., Geminate recombination in excited-state proton-transfer reactions: Numerical solution of the Debye-Smoluchowski equation with backreaction and comparison with experimental results. J. Chem. Phys. 1988, 88, 5620.

Walker, et al. - Proton Transfer in HPTS - Page 33 
27. Pines, E.; Huppert, D., Geminate recombination proton transfer reactions. Chem. Phys. Lett. 1986, 126,88 .

28. Chiariello, M. G.; Rega, N., Exploring Nuclear Photorelaxation of Pyranine in Aqueous Solution: an Integrated Ab-Initio Molecular Dynamics and Time Resolved Vibrational Analysis Approach. J. Phys. Chem. A 2018, 122, 2884.

29. Chiariello, M. G.; Raucci, U.; Donati, G.; Rega, N., Water-Mediated Excited State Proton Transfer of Pyranine-Acetate in Aqueous Solution: Vibrational Fingerprints from Ab Initio Molecular Dynamics. J. Phys. Chem. A 2021, 125, 3569.

30. Thomaz, J. E.; Walker, A. R.; Van Wyck, S. J.; Meisner, J.; Martinez, T. J.; Fayer, M. D., Proton Transfer Dynamics in the Aprotic Proton Accepting Solvent 1-Methylimidazole. J. Phys. Chem. B 2020, 124, 7897.

31. Kästner, J.; Sherwood, P., Superlinearly converging dimer method for transition state search. J. Chem. Phys. 2008, 128, 014106.

32. Bayly, C. I.; Cieplak, P.; Cornell, W.; Kollman, P. A., A well-behaved electrostatic potential based method using charge restraints for deriving atomic charges: the RESP model. J. Phys. Chem. 1993, 97, 10269.

33. Wang, J.; Wolf, R. M.; Caldwell, J. W.; Kollman, P. A.; Case, D. A., Development and testing of a general amber force field. J. Comp. Chem. 2004, 25, 1157.

34. Huige, C. J. M.; Altona, C., Force field parameters for sulfates and sulfamates based on ab initio calculations: Extensions of AMBER and CHARMm fields. J. Comp. Chem. 1995, 16, 56.

35. Jorgensen, W. L.; Chandrasekhar, J.; Madura, J. D.; Impey, R. W.; Klein, M. L., Comparison of simple potential functions for simulating liquid water. J. Chem. Phys. 1983, 79, 926.

36. Salomon-Ferrer, R.; Götz, A. W.; Poole, D.; Le Grand, S.; Walker, R. C., Routine Microsecond Molecular Dynamics Simulations with AMBER on GPUs. 2. Explicit Solvent Particle Mesh Ewald. J. Chem. Theory Comput. 2013, 9, 3878.

37. D.A. Case, V. B., J.T. Berryman, R.M. Betz, Q. Cai, D.S. Cerutti, T.E. Cheatham, III, T.A. Darden, R.E. Duke, H. Gohlke, A.W. Goetz, S. Gusarov, N. Homeyer, P. Janowski, J. Kaus, I. Kolossváry, A. Kovalenko, T.S. Lee, S. LeGrand, T. Luchko, R. Luo, B. Madej, K.M. Merz, F. Paesani, D.R. Roe, A. Roitberg, C. Sagui, R. Salomon-Ferrer, G. Seabra, C.L. Simmerling, W. Smith, J. Swails, R.C. Walker, J. Wang, R.M. Wolf, X. Wu and P.A. Kollman The Amber 
Molecular Dynamics Package, University of California: San Francisco, http://ambermd.org/, 2014.

38. Loncharich, R. J.; Brooks, B. R.; Pastor, R. W., Langevin Dynamics of Peptides: The Frictional Dependence of Isomerization Rates of $\mathrm{N}$-acetylalanyl-N'-methylamide. Biopolym. 1992, 32, 523.

39. Ufimtsev, I. S.; Martinez, T. J., Quantum Chemistry on Graphical Processing Units. 3. Analytical Energy Gradients, Geometry Optimization, and First Principles Molecular Dynamics. J. Chem. Theory Comput. 2009, 5, 2619.

40. Titov, A. V.; Ufimtsev, I. S.; Luehr, N.; Martinez, T. J., Generating Efficient Quantum Chemistry Codes for Novel Architectures. J. Chem. Theory Comput. 2013, 9, 213.

41. Eastman, P.; Swails, J.; Chodera, J. D.; McGibbon, R. T.; Zhao, Y.; Beauchamp, K. A.; Wang, L.-P.; Simmonett, A. C.; Harrigan, M. P.; Stern, C. D.; Wiewiora, R. P.; Brooks, B. R.; Pande, V. S., OpenMM 7: Rapid Development of High Performance Algorithms for Molecular Dynamics. PLOS Comput. Biol. 2017, 13, e1005659.

42. Tomasi, J.; Mennucci, B.; Cammi, R., Quantum Mechanical Continuum Solvation Models. Chem. Rev. 2005, 105, 2999.

43. Liu, F.; Sanchez, D. M.; Kulik, H. J.; Martinez, T. J., Exploiting graphical processing units to enable quantum chemistry calculation of large solvated molecules with conductor-like polarizable continuum models. Int. J. Quantum Chem. 2019, 119, e25760.

44. Liu, F.; Luehr, N.; Kulik, H. J.; Martinez, T. J., Quantum Chemistry for Solvated Molecules on Graphical Processing Units Using Polarizable Continuum Models. J. Chem. Theory Comput. 2015, $11,3131$.

45. Grimme, S.; Antony, J.; Ehrlich, S.; Krieg, H., A consistent and accurate ab initio parametrization of density functional dispersion correction (DFT-D) for the 94 elements H-Pu. $J$. Chem. Phys. 2010, 132, 154104.

46. Grimme, S.; Ehrlich, S.; Goerigk, L., Effect of the damping function in dispersion corrected density functional theory. J. Comp. Chem. 2011, 32, 1456.

47. König, P. H.; Ghosh N Fau - Hoffmann, M.; Hoffmann M Fau - Elstner, M.; Elstner M Fau Tajkhorshid, E.; Tajkhorshid E Fau - Frauenheim, T.; Frauenheim T Fau - Cui, Q.; Cui, Q., Toward theoretical analysis of long-range proton transfer kinetics in biomolecular pumps. $J$. Phys. Chem. A 2006, 110, 548.

Walker, et al. - Proton Transfer in HPTS - Page 35 
48. Chakrabarti, N.; Tajkhorshid, E.; Roux, B.; Pomes, R., Molecular Basis of Proton Blockage in Aquaporins. Structure 2004, 12, 65.

49. Taraphder, S.; Maupin, C. M.; Swanson, J. M. J.; Voth, G. A., Coupling Protein Dynamics with Proton Transport in Human Carbonic Anhydrase II. J. Phys. Chem. B 2016, 120, 8389.

50. Decornez, H.; Drukker, K.; Hammes-Schiffer, S., Solvation and Hydrogen Bonding Effects on Proton Wires. J. Phys. Chem. A 1999, 103, 2891.

51. Vuilleumier, R.; Borgis, D., Quantum Dynamics of an Excess Proton in Water Using an Extended Empirical Valence-Bond Hamiltonian. J. Phys. Chem. B 1998, 102, 4261.

52. Laage, D.; Elsaesser, T.; Hynes, J. T., Water Dynamics in the Hydration Shells of Biomolecules. Chem. Rev. 2017, 117, 10694.

53. Bekçioğlu, G.; Hoffmann, F.; Sebastiani, D., Solvation-Dependent Latency of Photoacid Dissociation and Transient IR Signatures of Protonation Dynamics. J. Phys. Chem. A 2015, 119, 9244.

Walker, et al. - Proton Transfer in HPTS - Page 36 